\section{A rapid, cost-effective method for counting human embryonic stem cell numbers as clumps}

Andrew B.J. Prowse, Ernst J. Wolvetang, and Peter P. Gray Australian Institute for Bioengineering and Nanotechnology, The University of Queensland, St Lucia, Australia

BioTechniques 47:599-606 (July 2009) doi 10.2144/000113151

Keywords: human embryonic stem cells; propidium iodide; high throughput; cell count; fluorescence

Enumeration of human embryonic stem cell (hESC) numbers through single cell digestion can be time consuming especially in high-throughput or multifactorial analysis containing $50+$ samples. We have developed a reproducible, cost-effective method of counting hESCs in clumps circumventing the need to manually dissociate each sample to single cells. The method is based on the DNA binding capacity of propidium iodide (PI) and subsequent fluorescent signal detection. Standard curves generated for cell numbers versus PI fluorescence as single cells or clumps showed an almost identical relationship in the lines of best fit. The reproducibility of the assay was first demonstrated by seeding hESC clumps at specific cell densities ranging $0.05-2 \times 10^{5}$ cells/well and then secondly by using the assay to count cell numbers after different growth conditions. Validation tests showed that consistent seeding densities are important in maintaining undifferentiated hESC culture and that the assay can be used to estimate relative cell numbers and growth curves with high accuracy.

\section{Introduction}

Human embryonic stem cells (hESCs) have the potential to differentiate into many cell types, making them ideal candidates for therapeutic applications. The earliest methods of hESC culture were labor intensive, time consuming, and did not lend themselves to scale-up. The evolution of hESC culture techniques has seen a move from manual passage of hESC colony clumps to enzymatic digestion and routine plating of clumps or single cells (1-4). In order to produce the number of cells required for downstream differentiation experiments and subsequent therapeutic applications (on the order of $10^{9}$ cells), culture methods are being developed that permit rapid cell expansion in an almost completely automated process $(5,6)$. These automated cell culture systems generally use split ratios rather than absolute cell number in sub-culturing protocols. However, the cell density of seeding, as well as the clump size, is critically important for maintaining both undifferentiated growth and controlled differentiation of hESCs, with both sparselyand too-densely-seeded hESCs having a propensity for slow growth or increased differentiation (7-9). Subculturing of cells as clumps then generates the issue of how to accurately determine seeding density based on the overall cell number. Treating a small sample of clumps with a dissociating agent such as trypsin or cell dissociation buffer followed by cell counting on a hemocytometer is feasible for a small number of samples. However, in higher-throughput analysis of hESCs, upwards of 50 individual culture conditions are examined, making manual dissociation and subsequent cell counting both time-consuming and laborintensive. Here, we demonstrate a method of counting total cell numbers in a 96-well plate format based on the DNA binding capacity of propidium iodide (PI) that avoids dissociating cell clumps. PI intercalates almost indiscriminately between DNA strands and once bound to DNA, its fluorescence is increased 20-30-fold. This method is reliable and cheap, allows for a rapid estimation of total cell numbers per well for many samples at once, and removes operator variability in cell counting. This cell-counting method will be useful for large scale automated culture of multiple samples $(5,6,10)$ where specific, expensive imaging technology (11) is not easily applicable.

\section{Materials and Methods}

Tissue culture

All reagents were from Invitrogen (Carlsbad, CA,USA) unless otherwise stated.hESClines MEL1 and MEL2 (Chemicon, Millipore, Billerica, MA, USA) were cultured on mouse embryonic fibroblasts (MEFs, 14,000/ $\mathrm{cm}^{2}$ ) in DMEM-F12 with L-glut supplemented by $20 \%$ knockout serum replacement (KOSR), $1 \times$ non-essential amino acids and $90 \mu \mathrm{M}$ $\beta$-mercaptoethanol. Cells were passaged in clumps using $200 \mathrm{U} / \mathrm{mL}$ collagenase IV. Differentiation controls were used in all experiments consisting of MEL1 or MEL2 grown in the media described above without feeders or maintenance factors.

Preparation of single cells

for standard curve

hESCs were harvested as clumps as described above and washed twice with PBS (without $\mathrm{Ca}^{2+}$ or $\mathrm{Mg}^{2+}$ ) before addition of TrypLE Express to dissociate cells. Cells were gently aspirated to dissociate. Cell counts were performed using a standard hemocytometer and cells resuspended in PBS at a concentration of $2 \times 10^{5}$ cells $/ 150 \mu \mathrm{L}$. Cells were transferred to a black-walled 96-well plate (Greiner Bio-One, Monroe, NC, USA) from $0-2 \times 10^{5}$ cells/well and final volumes adjusted to $150 \mu \mathrm{L} /$ well.

Preparation of cell clumps

for standard curve

hESC colonies were washed twice in warm PBS (without $\mathrm{Ca}^{2+}$ or $\mathrm{Mg}^{2+}$ ) before the addition of dispase $(1 \mathrm{mg} / \mathrm{mL}$; SigmaAldrich, St. Louis, MO, USA). Cells were incubated for $7 \mathrm{~min}$ and colonies broken into clumps using gentle pipetting. Suspensions were gently inverted several times to evenly distribute clumps immediately before dispensing 2 equal volumes from the center of the tube into separate tubes. One sample was treated with TrypLE Express to dissociate clumps into single cells and a cell count performed that represented cell numbers both in the single cell and clump suspensions. Equal clump size was determined using microscopy. Next, both the single cell and clump suspensions were centrifuged at $200 \times$ $g$ for 3 min. Both samples were resuspended in PBS at $2 \times 10^{5}$ cells $/ 150 \mu \mathrm{L}$. Both samples, having been derived from the same original suspension, were assumed to have the same total cell numbers. Cells were transferred (as described in section "Preparation of single cells for standard curve") to black-walled, 96-well plates in cell numbers ranging 
A

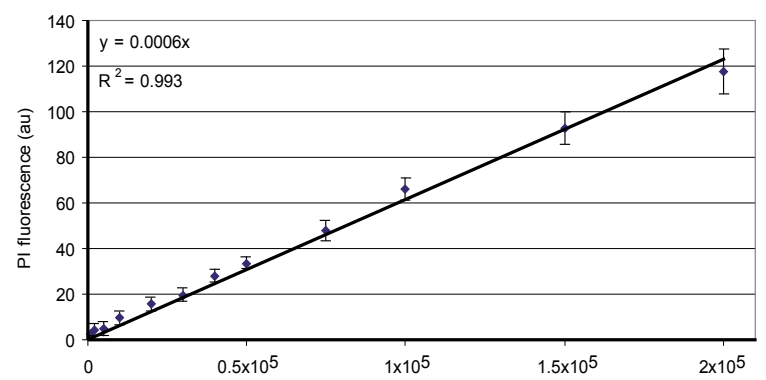

B

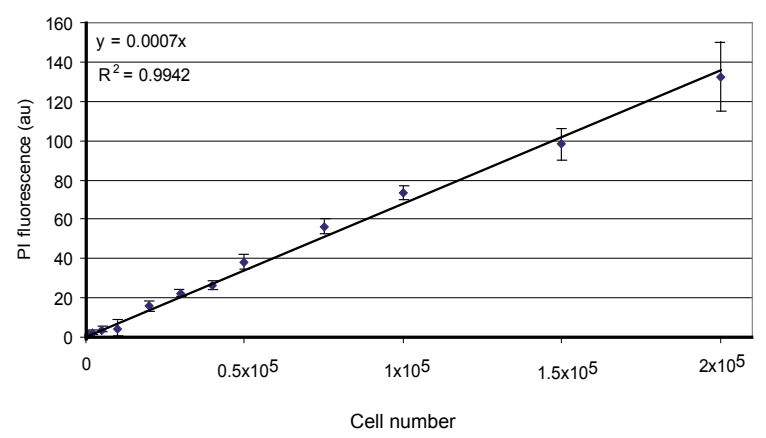

Figure 1. Standard curves for cell number versus $\mathrm{PI}$ fluorescence. hESC single cell suspensions (A) or clumps (B) were incubated with PI and Triton X-100 for 25 min before detection of PI fluorescence at $485 \mathrm{~nm}$ em/612 nm ex. Error bars are \pm SEM $(n=6$, Figure $1 \mathrm{~A}$ or $n=3$, Figure $1 \mathrm{~B}$ ) of independent experiments. The equation and $R^{2}$ values of the line of best fit are shown in the top left corner of each graph.

$0-2 \times 10^{5}$ cells/well and final volumes adjusted to $150 \mu \mathrm{L} /$ well.

Determination of cell number using propidium iodide (PI)

A $4 \times$ stock solution of $40 \mu \mathrm{g} / \mathrm{mL}$ PI and $0.4 \%$ Triton X-100 in PBS was made immediately following transfer of cells to a 96-well plate. A multi-channel pipet (Cat. no. EW-24553-07; Eppendorf South Pacific, North Ryde, NSW, Australia) was used to dispense $50 \mu \mathrm{L}$ stock solution to each well containing $150 \mu \mathrm{L}$ cell suspension, giving a final working concentration of $10 \mu \mathrm{g} / \mathrm{mL}$ PI and $0.1 \%$ Triton $\mathrm{X}-100$. PI fluorescence based on binding of dsDNA was measured using a SpectraMax M5 fluorometer (Molecular Devices, Sunnyvale, CA, USA) at an excitation of $485 \mathrm{~nm}$, emission of $612 \mathrm{~nm}$, and a cutoff of $610 \mathrm{~nm}$. Cells were incubated at room temperature for $1 \mathrm{~h}$ with measurements made from the top of the plate every $5 \mathrm{~min}$. Blanks contained $150 \mu \mathrm{L}$ PBS and $50 \mu \mathrm{L}$ $4 \times$ stock solution. Data was analyzed and standard curves generated using SoftMax Pro v5.01 (Molecular Devices).

Plating hESC at varying densities MEL1 and MEL2 cells were dissociated into clumps as previously outlined and a cell count performed on triplicate $150-\mu \mathrm{L}$ aliquots of each cell line using the Spectramax fluorometer. PI fluorescence signals obtained were used to estimate cell number using the standard curve and equation $y=7 \times 10^{-4} x$. Cells were plated in 12-well plates containing glass coverslips coated with Matrigel $(34.7 \mu \mathrm{g} /$ $\mathrm{cm}^{2 ;} \mathrm{BD}$, Franklin Lakes, NJ, USA) at densities ranging $0.05-2 \times 10^{5}$ cells/well and cultured for 10 days. mTESR medium (Stem Cell Technologies, Melbourne, Australia) was changed daily.

\section{Evaluation of hESC plurpotency} markers after different seeding densities MEL1 and MEL2 were analyzed by observation of the pluripotency markers GCTM2 and CD9 using immunofluorescence and flow cytometry. For immunofluorescence, cells were fixed with cold ethanol for $30 \mathrm{~min}$. After each step, cells were washed twice in TRIS-buffered saline with $0.05 \%$ Tween-20. Cells were permeabilized with $0.1 \%$ Triton X-100 then blocked for $15 \mathrm{~min}$ in KOSR. Cells were stained with CD9 antibody $(2.5 \mu \mathrm{g} / \mathrm{mL}$; The Australian Stem Cell Centre, Melbourne, Australia) for $30 \mathrm{~min}$ and then anti-mouse $\operatorname{IgG}$ Alexafluor $488(1: 2000,1 \mu \mathrm{g} / \mathrm{mL})$ in the dark for $30 \mathrm{~min}$, counterstained with 0.1 $\mu \mathrm{g} / \mathrm{mL}$ Dapi, and mounted with anti-fade solution. A mouse IgG isotype control was used to determine nonspecific background staining. Cells were visualized using an LSM 510 META on an AxioObserver using Z1 and Zen 2007 Light Edition SP1 software (Carl Zeiss, Jena, Germany). Flow cytometry for CD9 and GCTM2 $(2.5 \mu \mathrm{g} / \mathrm{mL})$ was performed on $10^{6} \mathrm{cell} / \mathrm{s} / \mathrm{mL}$ as previously described (12). Detection was

\section{BioTechnioues}

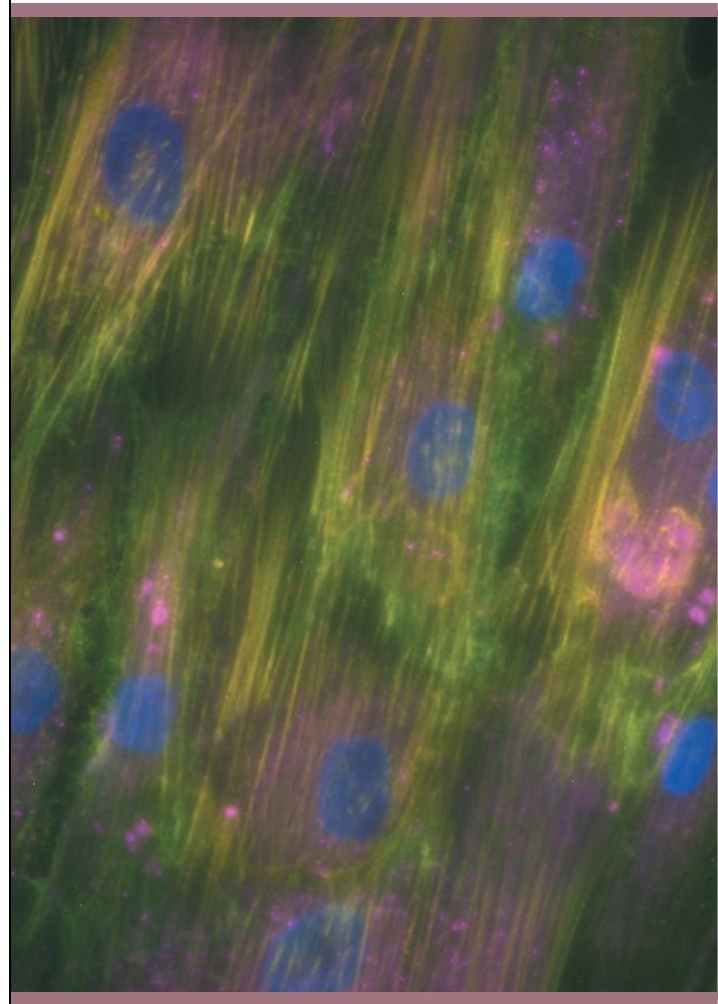

\section{Free Subscription}

BioTechniques is the first peer-reviewed journal with open access to the whole of the life science community.

$100 \%$ life science

$100 \%$ peer-reviewed methods

$100 \%$ "everyday" practical information

Sign up for free at

www.BioTechniques.com/subscribe

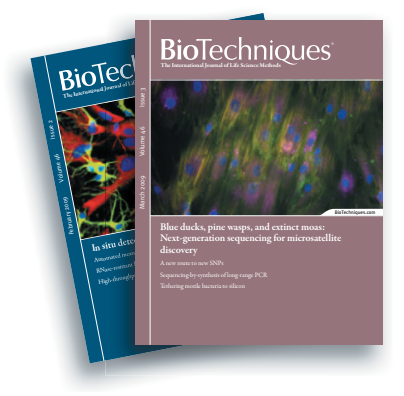




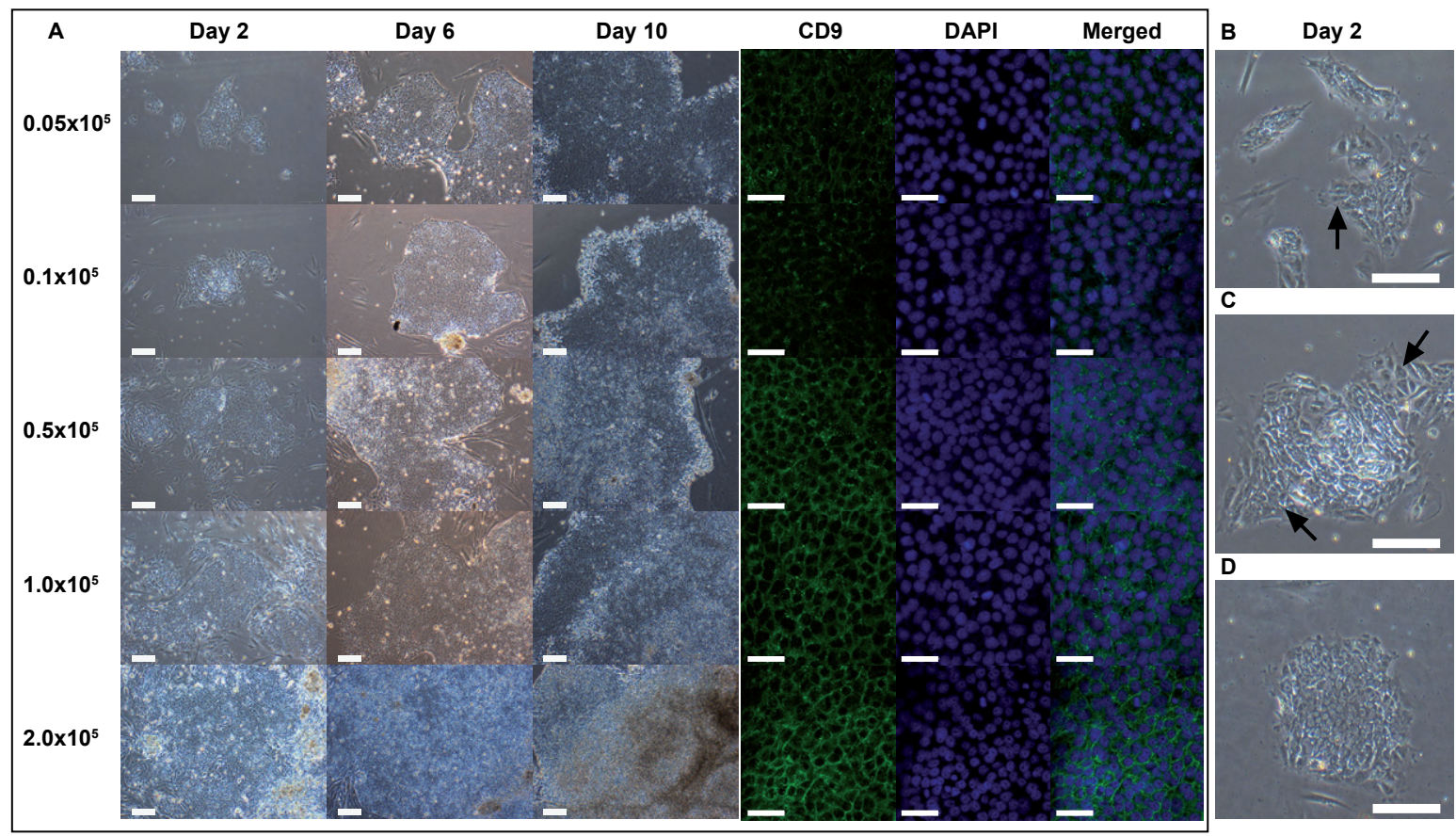

Figure 2. Morphological and cell surface marker expression analysis in MEL1. Images demonstrate reproducible plating of cells at different densities. Cells were grown for 10 days in mTESR media. (A) Phase contrast and immunofluorescent images of MEL1 grown at different densities. Columns for phase contrast are arranged according to days in culture. For immunofluorescence at day 10, columns are, from left to right: CD9; DAPI; merged image. Rows are arranged according to seeding density. Images (B) and (C) show differentiated cell morphology at $0.05 \times 10^{5}$ and $0.1 \times 10^{5}$ cells/well on day 2 (arrows). (D) Typical undifferentiated cell morphology at $0.5 \times 10^{5}$ cells/well on day 2 . Scale bar for phase contrast images is $200 \mu \mathrm{m}$ and $50 \mu \mathrm{m}$ for immunofluorescence images. MEL2 showed similar staining patterns (data not shown).

\section{When superior perfo
is the only option.}
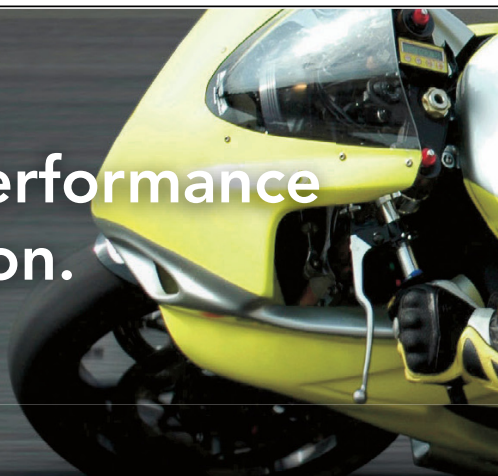

\section{(1)}


A

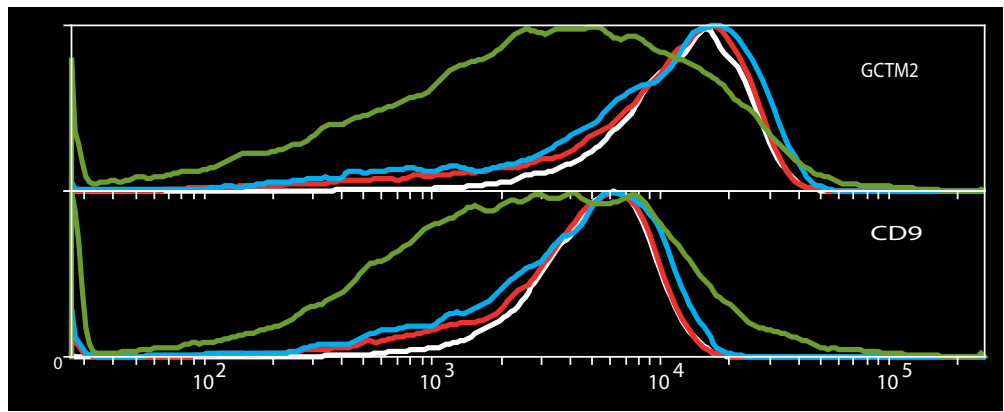

B

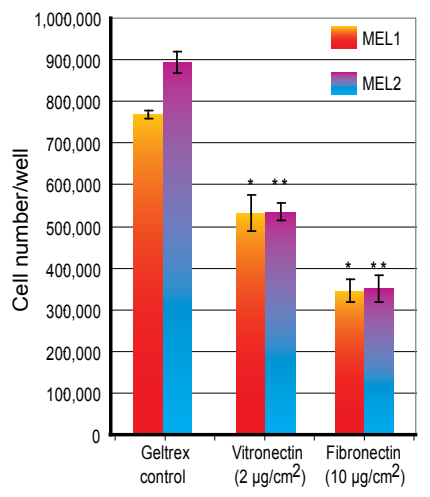

C

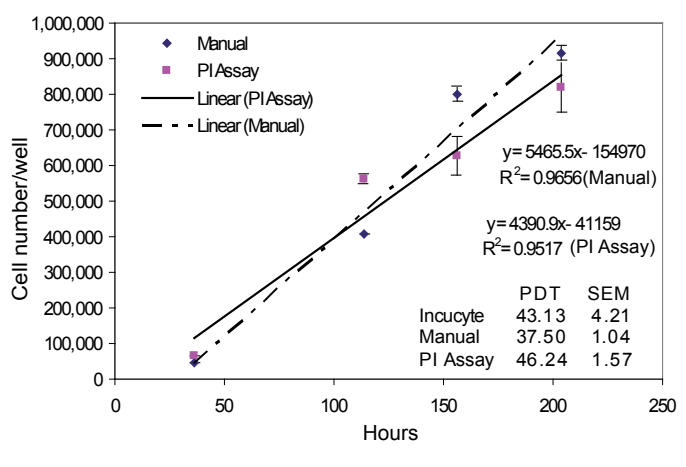

ECM and concentration

Figure 3. Endpoint pluripotency marker expression and cell number enumeration. (A) Histogram expression profile after 10 days culture for the pluripotency markers GCTM2 and CD9 in MEL1 seeded at the following densities: Green, differentiation control $\left(1 \times 10^{5}\right.$ cells/well); white, $0.05 \times 10^{5}$ cells/well; red, $0.1 \times 10^{5}$ cells/well; blue, $1 \times 10^{5}$ cells/well. (B) Final seeding densities of hESCs seeded at 0.5 $\times 10^{5}$ cells/well on different ECM proteins. At day 10 , total cells/well were enumerated using our $\mathrm{PI}$ assay. Error bars are \pm SEM of triplicate wells. ${ }^{*} P<0.05$ versus MEL1 Geltrex control; ${ }^{*} P<0.05$ versus MEL2 Geltrex control. (C) Growth curve of MEL2 cells grown on Geltrex counted at 4 different time points using standard hemocytometer counts and the PI assay. Population doubling times (PDT) for each method as well as the Automated Incucyte Cell Imager are displayed bottom right. Error bars are \pm SEM of triplicate wells.

carried out using AlexaFluor 633 anti-mouse IgM (GCTM2) and Alexafluor APC-750 anti-mouse IgG (CD9) $(1: 2000,1 \mu \mathrm{g} / \mathrm{mL}$ final concentration, $10^{6}$ cells $\left./ \mathrm{mL}\right)$. Appropriate mouse $\operatorname{IgM}$ and $\operatorname{IgG}$ isotype controls were used to set background fluorescence levels. Data was analyzed using WEASEL Software V2.4 (Walter and Eliza Hall Institute for Medical Research, Melbourne, Australia).

Determination of end point cell numbers and growth curves

MEL2 cells were seeded in clumps at $0.5 \times 10^{5}$ cells/well in a 12 -well plate. Dishes were coated with Geltrex (1:30 as per manufacturer's instructions) fibronectin (10 $\left.\mu \mathrm{g} / \mathrm{cm}^{2}\right)$, or vitronectin $\left(2 \mu \mathrm{g} / \mathrm{cm}^{2}\right)$. Cultures were grown in StemPro media over 9-10 days with daily media changes. Triplicate Geltrex-coated wells were harvested at days $2,5,7$, and 9 as clumps using collagenase IV $(200 \mathrm{U} / \mathrm{mL})$, or as single cells using TrypLE Express. Data was used to generate growth curves and population doubling times using a hemocytometer or the PI assay. Replicate extracellular matrix (ECM)-coated wells were harvested with collagenase IV on day 9 and cells were counted using the PI assay to determine end point numbers. An Incucyte cell imager was used in parallel (Essen Instruments, Ann Arbor, MI, USA), according to manufacturer's instructions.

\section{Results and discussion}

To circumvent the time- and labor-intensive manual counting of hESCs prior to plating, we opted to use the DNA content of hESCs as a measure of total cell number. To first establish the relationship between hESC number and PI fluorescence, we seeded increasing amounts of manually counted single hESCs in microtiter plates and PI stained them in a one-step procedure through addition of a $4 \times$ stock solution of PI/Triton X-100 in PBS.

As shown in Figure 1A, there was a linear relationship between PI fluorescence and hESC number when using $<2 \times 10^{5}$ cells per well of a microtiter plate. Since hESCs are often subcultured as clumps of varying sizes containing $20-100$ cells, we next determined the relationship between PI fluorescence and hESC cell number in clumps. One half of an equal distribution of hESC clumps was next dissociated into single cells and manually counted while increasing amounts of hESC clumps were seeded into microtiter plates and PI-stained using the protocol described above. As shown in Figure 1B, the relationship between PI fluorescence and hESC cell number was found to be almost identical to that for single cells (linear slope $y=6 \times 10^{-4} x$ versus $y=7 \times 10^{-4} x$, where $y$ is fluorescence and $x$ is cell number), suggesting that PI had stained the DNA with equal efficiency in clumps as in singlecell suspensions. Standard deviations for the highest cell counts performed $\left(2 \times 10^{5}\right)$ on clumps were \pm 30.6 fluorescence units equaling $\pm 44,000$ cells/well of a 96-well plate, using the equation in Figure 1B. To avoid further error in back-calculating total cells in original culture conditions, the most accurate means of performing the assay was to maintain $<2 \times 10^{5}$ cells within aliquots for analysis. This also allows for sufficient surplus cells for analysis or propagation.

To illustrate the efficacy of the rapid hESC enumeration procedure and to exemplify the importance of optimal seeding density for the maintenance of undifferentiated hESC growth, we seeded hESCs in clumps at varying cell densities of $0.05,0.1$, $0.5,1$, and $2 \times 10^{5} \mathrm{hESCs} /$ well (based on the PI hESC quantification method) and subsequently cultured these for 10 days. Figure $2 \mathrm{~A}$ demonstrates the density of MEL1 colonies at days 2, 6, and 10 that were plated at varying cell densities based on cell counts using the PI assay. MEL1 cells grew as a monolayer in every condition except the $2 \times 10^{5}$ cell seeding density, wherein overcrowding of the cells was evident at day 10. Early morphological analysis (day 2 after seeding) of the lower-density cultures $\left(0.05-0.1 \times 10^{5}\right.$ cells/well $)$ showed cells of an elongated or triangular morphology (Figure 2, B and C, black arrows) whereas cells seeded at higher densities $(\geq 0.5 \times$ $10^{5}$ ) displayed a typical round morphology with a high nucleus-to-cytoplasm ratio that is characteristic of undifferentiated hESCs (Figure 2D). Next, the hESCs were analyzed for the expression of the pluripotency marker CD9 by direct immunofluorescence and expression of both CD9 and GCTM2 by flow cytometry. In agreement with the apparent reduction in the fraction of undifferentiated hESCs by morphological criteria (Figure 2, B-D), the two lowest cell seeding densities $\left(0.05\right.$ and $0.1 \times 10^{5}$ cells $/$ well) showed reduced expression of CD9 
compared with the higher seeding densities $\left(0.5,1\right.$, and $2 \times 10^{5}$ cells/well, Figure $\left.2 \mathrm{~A}\right)$. In contrast, however, the CV for the spread of pluripotency markers was decreased for lower-seeded populations $\left(0.05 \times 10^{5}\right.$ cells/ well, GCTM2 CV $=54.5 \pm 1.9$, CD9 CV $=52.1 \pm 1.5)$ compared with higher-density cultures $\left(0.1 \times 10^{5}\right.$ cells/well, GCTM2 CV $=67.9 \pm 7, \mathrm{CD} 9 \mathrm{CV}=69.7 \pm 8.7)$ indicating a more uniform cell type in the lower cell densities. We hypothesize that seeding cells at low densities, while leading to increased differentiation (Figure 2, A-C), also selects for a subset of cells capable of survival that results in a purer population of undifferentiated cells with a narrower distribution of pluripotency markers (Figure 3A). In contrast, cells seeded at higher densities allow for the proliferation and survival of a larger subset of undifferentiated cells.

Although our data indicate that the PI assay can be used to accurately seed hESCs at different densities and highlight the importance of determining appropriate seeding densities for hESC culture, it should be noted that undifferentiated cell growth is dependent not only on cell numbers/well but also clump size (9) and that this varies between different culture systems and harvesting techniques. The counting method described here performs equally well on hESCs with various clump sizes (unpublished data) and thus will allow hESC culture optimization provided the clump size within a specific culture protocol is kept consistent.

To illustrate the power of the hESC enumeration assay described in this paper in high-throughput multifactorial analyses, we next seeded hESC at identical densities, based on our PI assay (verified with manual cell counting) on Geltrex- or ECM-coated wells and determined cell numbers after 10 days using the PI assay. Figure 3B shows that there was very little interwell variation in hESC cell numbers and that the PI assay was able to successfully differentiate between wells of different cell densities with high accuracy $(P<0.05$, Student's two-tailed $t$-test). Finally, to further demonstrate the power of the assay in an end point analysis, growth curves for MEL2 grown on Geltrex in StemPro were determined using three independent cell number determinants: manual hemocytometer counts, an Incucyte cell imager (Essen Instruments) and the PI assay. Figure 3C shows a comparative growth curve of the PI assay and the manual cell counts. The Incucyte growth curve data was not directly comparable to the other two methods as it is based on confluence rather than overall cell number. However, it was possible to directly compare population doubling times (PDT) determined by all three methods (Figure 3C). Although the PDT calculated using the automated cell imager was closer to the PDT determined using the hemocytometer cell counting method, there are a number of concerns using this type of automated analysis in hESC cultures. First, the errors are significantly greater than either the manual or PI cell counting methods $( \pm 4.21 \mathrm{~h}$ compared with \pm 1.04 and $\pm 1.57 \mathrm{~h}$, respectively). Second, the PDT is determined using percent confluency and not overall cell numbers, which, in heterogeneous hESC cultures that differentiate into cells with dramatically different surface area, can introduce further errors or overestimated PDTs. These and other automated systems (11) are limited by their ability to perform confluency measurements only on a two-dimensional (surface when it is well established that hESCs often grow in three-dimensional colonies. Thirdly, our method is cheap and does not require advanced instrumentation when compared with automated imaging systems.

Our data indicate that the assay performs well when compared with manual counting and has the added advantage of removing operator-mediated counting errors and judgment calls when scoring cell numbers with a hemocytometer. The assay requires access to a standard fluorescence plate reader and can in principle be adapted to the use of other DNA-binding fluorophores such as picogreen or Cy-5. We calculate that when using this assay it is possible to accurately enumerate the cell number of up to 96 hESC clump suspensions in $45 \mathrm{~min}$ (15 min pipetting $+30 \mathrm{~min}$ read) while the same outcome using a manual counting method would require over $8 \mathrm{~h}$ (dissociation $3 \mathrm{~min}$, hemocytometer counting $2 \mathrm{~min}=5 \mathrm{~min} ; 5$ $\min \times 96$ suspensions $=480 \mathrm{~min}=>8 \mathrm{~h}$. In each case, the time taken for harvesting the cells from the plates was not considered. Adoption of this cost-effective and rapid technique will allow for high-throughput screening of hESC numbers for relative end point analysis of cell proliferation, growth curves, and consistent seeding of cells.

\section{Acknowledgments}

We would like to thank Justin Cooper White and Michael Doran for supplying the ECM-coated surfaces, Kylie Mallitt for assistance with statistics, and the Australian Stem Cell Centre (Queensland Node) for supply of hESC lines. This research was made possible through a grant provided by the Australian Stem Cell Centre, Melbourne, Australia.

The authors declare no competing interests.

\section{References}

1. Thomson, J.A., J. Itskovitz-Eldor, S.S. Shapiro, M.A. Waknitz, J.J. Swiergiel, V.S. Marshall, and J.M. Jones. 1998. Embryonic stem cell lines derived from human blastocysts. Science 282:1145-1147.

2. Xu, C., M.S. Inokuma, J. Denham, K. Golds, P. Kundu, J.D. Gold, and M.K. Carpenter. 2001. Feeder-free growth of undifferentiated human embryonic stem cells. Nat. Biotechnol. 19:971-974.

3. Reubinoff, B.E., M.F. Pera, C.Y. Fong, A. Trounson, and A. Bongso. 2000. Embryonic stem cell lines from human blastocysts: somatic differentiation in vitro. Nat. Biotechnol. 18:399404.

4. Ludwig, T.E., V. Bergendahl, M.E. Levenstein, J. Yu, M.D. Probasco, and J.A. Thomson. 2006. Feeder-independent culture of human embryonic stem cells. Nat. Methods 3:637646.

5. Terstegge, S., I. Laufenberg, J. Pochert, S. Schenk, J. Itskovitz-Eldor, E. Endl, and O. Brustle. 2007. Automated maintenance of embryonic stem cell cultures. Biotechnol. Bioeng. 96:195-201.

6. Thomas, R.J., D. Anderson, A. Chandra, N.M. Smith, L.E. Young, D. Williams, and C. Denning. 2009. Automated, scalable culture of human embryonic stem cells in feeder-free conditions. Biotechnol. Bioeng. 102:16361644.

7. Draper, J.S., H.D. Moore, L.N. Ruban, P.J. Gokhale, and P.W. Andrews. 2004. Culture and characterization of human embryonic stem cells. Stem Cells Dev. 13:325-336.

8. Ng, E.S., R.P. Davis, L. Azzola, E.G. Stanley, and A.G. Elefanty. 2005. Forced aggregation of defined numbers of human embryonic stem cells into embryoid bodies fosters robust, reproducible hematopoietic differentiation. Blood 106:1601-1603.

9. Bauwens, C.L., R. Peerani, S. Niebruegge, K.A. Woodhouse, E. Kumacheva, M. Husain, and P.W. Zandstra. 2008. Control of human embryonic stem cell colony and aggregate size heterogeneity influences differentiation trajectories. Stem Cells 26:2300-2310.

10.Oh, S. and A.B. Choo. Advances and perspectives in human embryonic stem cell bioprocessing. Drug Discov. Today. Technol. (In press.)

11. Narkilahti, S., K. Rajala, H. Pihlajamaki, R. Suuronen, O. Hovatta, and H. Skottman. 2007. Monitoring and analysis of dynamic growth of human embryonic stem cells: comparison of automated instrumentation and conventional culturing methods. Biomed. Eng. Online 6:11.

12.Herszfeld, D., E. Wolvetang, E. Langton-Bunker, T.L. Chung, A.A. Filipczyk, S. Houssami, P. Jamshidi, K. Koh, et al. 2006. CD30 is a survival factor and a biomarker for transformed human pluripotent stem cells. Nat Biotechnol. 24:351-357.

Received 12 January 2009; accepted 11 March 2009.

Address correspondence to Ernst Wolvetang, Australian Institute for Bioengineering and Nanotechnology (AIBN), Corner College and Cooper Rds (Bldg 75), The University of Queensland, Brisbane Qld 4072, Australia. email: e.wolvetang@uq.edu.au 